\title{
BUENAS PRÁCTICAS Y NUEVAS TECNOLOGÍAS EN LA COOPERACIÓN PENAL EN EL MERCOSUR
}

\section{BOAS PRÁTICAS E NOVAS TECNOLOGIAS EM COOPERAÇÃO PENAL NO MERCOSUL}

Juan José Cerdeira ${ }^{\star}$

Resumen: El presente aborda las diferentes problemáticas que presenta la cooperación jurídica en materia penal desde la perspectiva internacional del espacio MERCOSUR y Estados Asociados y la importancia que revisten las buenas prácticas y las nuevas tecnologías para el cabal desenvolvimiento de dicha cooperación internacional como instrumento de combate contra el crimen. En el desarrollo se expondrán los desafíos que se presentan actualmente en el marco regional, atento sus características como esquema de integración y seguidamente se expondrán algunas propuestas y soluciones como aporte.

Resumo: Este artigo discute os diferentes problemas apresentados pela cooperação jurídica em questões criminais a partir da perspectiva internacional do espaço MERCOSUL e dos Estados Associados e a importância das boas práticas e das novas tecnologias para o pleno desenvolvimento dessa cooperação internacional como instrumento de combate ao crime. O desenvolvimento apresentará os desafios atualmente apresentados no quadro regional, atento às suas características como um esquema de integração e, em seguida, expôs algumas propostas e soluções.

Palabras clave: Cooperación Penal, MERCOUR, Tecnologías

Palavras-chave: Cooperação criminal, MERCOUL, Tecnologías

\section{INTRODUCCIÓN}

El presente aborda el desarrollo que ha tenido la asistencia jurídica penal en el ámbito del MERCOSUR, los pasos recorridos, los resultados obtenidos y los desafíos pendientes en la lucha contra el crimen.

* Universidad de Buenos Aires, Argentina.

E-mail: juanjocer@hotmail.com

Recibido: 15/09/2017. Aceptado: 10/10/2017. 
El análisis se planteará desde la perspectiva de la República Argentina, en tanto inserto en el esquema mercosureño, con miras a brindar respuestas a las demandas que se producen en torno a la materia en el contexto regional.

Durante el desarrollo se verá de qué manera en el marco de diferentes foros del esquema regional intergubernamental se promueve la utilización de los instrumentos y mecanismos jurídico institucionales más propicios para la cooperación y a la instauración de las buenas prácticas y el uso de la tecnología como herramientas novedosas que procuran garantizar la sanción al delito y a la vez los derechos de las víctimas en su carácter de más vulnerables.

El propósito es garantizar un efectivo acceso a la justicia y a soluciones rápidas y eficaces que les permitan facilitar la cooperación más allá de sus fronteras nacionales.

La temática es un tema de suma relevancia y sensibilidad ya que en muchos casos se tratará de planteos jurídicos complejos.

Se pasará revista a las actividades que despliegan los distintos foros con incumbencias en el tema dentro de la estructura institucional del MERCOSUR y también la recepción que hacen los Estados de la región de las soluciones alcanzadas a nivel regional, quienes a través de las políticas públicas o a través de Autoridades Centrales o Coordinadoras promueven el desarrollo de diferentes mecanismos que les permiten avanzar en la lucha contra el crimen desde una perspectiva armónica.

\section{LA COOPERACIÓN INTERNACIONAL EN EL MERCOSUR}

Desde la perspectiva regional del MERCOSUR y sus Estados Asociados, hay distintos aspectos de la cooperación penal que ameritan ser especialmente considerados, atento las particulares características que se han instrumentado desde ese espacio para el combate de la criminalidad ${ }^{1}$.

Uno de ellos es el del papel de las Autoridades Centrales cuyo rol es fundamental ya que se trata de territorios que son muy dilatados y muchas veces los jueces no siempre pueden acceder fácilmente al conocimiento de todas las fuentes, o al complejo entramado jurídico necesario al momento de aplicación de ellas. Entonces, se hace necesaria la intervención de alguna institución estatal que sea de nexo y que le informe, las formas y procedimientos a seguir.

Por lo tanto, cabe resaltar la importancia que se le diera al tema de las Autoridades Centrales en el ámbito del MERCOSUR, ya que en el año 1996 con el Protocolo de San Luis ${ }^{2}$ y en los Protocolos y Acuerdos

1 BOGGIANO, Antonio. Derecho Penal Internacional. Buenos Aires. Lexis Nexis- Abeledo Perrot, 2003 y 2006.

2 MERCOSUR. Protocolo de Asistencia Jurídica Mutua en Asuntos Penales entre los Estados Parte del MERCOSUR. Aprobado por Decisión CMC N 2/96 
en materia de Asistencia Penal que le siguieron posteriormente, se hizo hincapié en la comunicación clara, frecuente y fluida entre Autoridades Centrales como uno de los medios más eficaces para el funcionamiento de la cooperación, sobre todo en la materia penal. Más recientemente los contemplan otros instrumentos de cooperación penal como el "Acuerdo Marco para la Creación de Equipos Conjuntos de Investigación ${ }^{3 "}$ o el "Acuerdo Orden MERCOSUR de Detención y Procedimientos de Entrega", elaborado todos ellos en el marco de la Reunión de Ministros de Justicia ${ }^{5}$.

En otro orden de ideas y como buena práctica, resulta interesante referirse a la tarea desarrollada en diferentes foros especializados en los que se está trabajando en la capacitación de jueces en materia internacional como forma de contribuir a la difusión y mejor aplicación de la nueva normativa.

En cuanto a la problemática de la cantidad de convenciones aplicables es una cuestión aparte que merece especial atención., en tanto hay demasiadas fuentes bilaterales, regionales, multilaterales., sobre los mismos temas entre las mismas partes.

Eso sumado a que en este espacio regional los países poseen territorios extendidos, los jueces a veces no pueden tomar conocimiento de todas las fuentes que se van sucediendo porque no hay suficientes capacitación, difusión y seminarios, jornadas, simposios que se ocupen del tema.

Por otra parte, las autoridades judiciales, muchas veces carecen del fluido contacto que debieran tener con las Autoridades Centrales, de lo que resulta un consecuente desconocimiento de las normas. Se torna pues necesario, que el juez, al momento de aplicar algunos de estos convenios o algunas de estas leyes, deba averiguar si está vigente en su país, si lo está en el Estado requirente o requerido, según el caso, si rige con el otro país, etc.

No menos relevante es el destacar que en los últimos años, se han venido desarrollando nuevos institutos jurídicos que ayudan a la lucha contra el crimen. Es el caso, por ejemplo, de los programas como el de "protección de víctimas y testigos"; o figuras como el "arrepentido", "testigo protegido" o el "agente encubierto"

Con relación a las cláusulas de preservación de la seguridad nacional y del orden público, se está debatiendo últimamente la problemática que este tipo de normas genera para la efectividad de la cooperación, al ser utilizados como causales para denegar la asistencia

3 MERCOSUR. Decisión N²2/10. Acuerdo Marco de Cooperación entre los Estados Parte del MERCOSUR y Estados Asociados.

4 MERCOSUR, Decisión CMC N 48/10: Acuerdo sobre Orden de Detención y Procedimientos de Entrega entre los Estados Parte del MERCOSUR y Asociados

5 Disponible en <www.mercosur.int $>$

6 BAIGÚN, David. "El abogado y las buenas causas". Revista del Colegio Público de Abogados de la Capital Federal. 2004, n4. 
solicitada o en su caso la extradición.

Los Estados, en general suelen aferrarse a este tipo de normas y esto también ha empezado a cuestionarse, por lo menos en cuanto a su postura estricta y se ha notado la dificultad que puede ocasionar la aplicación rígida de estos principios para el logro de los cometidos últimos de la cooperación.

Por eso es necesario generar nuevas ideas para la solución de ese tipo de barreras y agudizar la imaginación de los especialistas con miras a aportar novedosos elementos o herramientas que ayuden a la despolitización de estos conceptos y evitar de esa manera que esos delincuentes que trasponen las fronteras, busquen el amparo de una legislación más protectora para eludir la punición de sus ilícitos.

A continuación se esquematizará un listado de varias de las dificultades detectadas en la cooperación y el consiguiente desafío que ello implica.

\begin{tabular}{|l|l|}
\hline \multicolumn{1}{|c|}{ 1. Dificultad a considerar } & \multicolumn{1}{c|}{ 2. Desafío } \\
\hline $\begin{array}{l}\text { Entrada en Vigencia de Normas de fuente } \\
\text { interna y convencional }\end{array}$ & $\begin{array}{l}\text { Diferentes tipos de incorporación y } \\
\text { consecuentes demoras en la entrada en } \\
\text { vigencia. }\end{array}$ \\
\hline $\begin{array}{l}\text { Proliferación de Fuentes Convencionales } \\
\text { sobre mismos temas entre mismas } \\
\text { Partes. }\end{array}$ & $\begin{array}{l}\text { Falta de difusión. Superposición de } \\
\text { textos. Complicación en su aplicación } \\
\text { por parte de operadores de la justicia. }\end{array}$ \\
\hline Cláusulas en beneficio de la cooperación & $\begin{array}{l}\text { Mantiene vigentes todos los tratados } \\
\text { y dificulta la identificación del más } \\
\text { efectivo. }\end{array}$ \\
\hline Territorios muy extendidos. & $\begin{array}{l}\text { Distancia entre operadores del } \\
\text { derecho y las Autoridades Centrales o } \\
\text { Coordinadoras. }\end{array}$ \\
\hline $\begin{array}{l}\text { Necesaria interconexión entre } \\
\text { operadores de justicia }\end{array}$ & $\begin{array}{l}\text { Casi nula. Exceso de celo en las áreas de } \\
\text { su incumbencia. Trabajo en equipo. }\end{array}$ \\
\hline $\begin{array}{l}\text { Cooperación institucional horizontal } \\
\text { por sectores o carteras de incumbencias. }\end{array}$ & $\begin{array}{l}\text { Vinculación “intra país” y con los otros } \\
\text { Estados. }\end{array}$ \\
\hline $\begin{array}{l}\text { Necesidad de Estadísticas, Bancos de } \\
\text { Datos e Intercambio de Información. }\end{array}$ & $\begin{array}{l}\text { Información sectorizada y } \\
\text { descoordinada entre las fuentes } \\
\text { productoras. }\end{array}$ \\
\hline Recursos presupuestarios escasos & $\begin{array}{l}\text { Falta de medios para informatizar } \\
\text { sistemas, bancos de datos y promoción y } \\
\text { utilización de los medios informáticos. }\end{array}$ \\
\hline
\end{tabular}

Se impone entonces un desarrollo de las dificultades apuntadas y los desafíos que ellos implican: 


\section{a) Entrada en vigencia}

La entrada en vigencia de las fuentes internas en materia penal, suelen preceder de un largo período de análisis, dictámenes y discusión en los ámbitos nacionales. Desde la perspectiva argentina, suele haber varias carteras involucradas en su debate, el análisis de las características procesales de sus postulados y el consiguiente cuidado que merecen en atención al carácter federal de nuestro ordenamiento.

Por su parte, las fuentes bilaterales o multilaterales que se concluyen deben seguir un procedimiento de negociación, firma, aprobación legislativa y eventual ratificación o adhesión que demandará también un largo tiempo, muchas veces años, para su efectiva entrada en vigencia ${ }^{7}$.

Para el caso de los Protocolos y Acuerdos Mercosur, por ejemplo, es necesaria la aprobación legislativa de todos los Miembros Plenos, el depósito de los respectivos instrumentos de ratificación y finalmente con la notificación del depósito del último, a los 30 días entra en vigencia para el bloque regional ${ }^{8}$.

\section{b) Proliferación legislativa}

Como consecuencia de lo referido anteriormente, conviven en la materia, convenios de cooperación bilaterales, regionales y multilaterales, en muchos casos, que versan sobre las mismas materias, entre los mismos Estados. Por ejemplo, i) el Tratado de Montevideo de Derecho Penal de 1889; ii) las Convenciones Interamericanas; iii) los Protocolos y Acuerdos MERCOSUR; iv) los Convenios Iberoamericanos; v) las Convenciones de la $\mathrm{ONU}_{2}$ vi) muchos bilaterales en la materia entre los mismos países y sobre los mismos temas.

\section{c) Cláusulas de compatibilidad y en beneficio de la cooperación}

Entre las consecuencias sobrevinientes de la proliferación antes referida está la que se utiliza para evitar que se queden sin regulación en los nuevos convenios que se suscriben, cuestiones ya resueltas en otros que existen sobre la materia Ello provoca que la técnica legislativa

7 HARDING, Christopher. "Exploring the intersection of European law and national criminal law”. European Law Review. 2000, vol 25/4.

8 BOLDORINI, María.Cristina. y CZAR de ZALDUENDO, Susana. "La estructura jurídicoinstitucional del MERCOSUR después del Protocolo de Ouro Preto". En Boletín Informativo, $1995, n^{\circ} 283$, p.73-90.

9 a) Conferencia de Ministros de Justicia de los Países Iberoamericanos. a) Proyecto de Tratado Iberoamericano sobre Transmisión de Solicitudes de Cooperación Jurídica entre Autoridades Centrales); b) Tratado constitutivo de la IberRed (Red Iberoamericana de Cooperación Jurídica Internacional), y otros de cooperación jurídica utilizables. 
ensayara un tipo de fórmula que pretende facilitar la operatoria pero que en muchos casos puede llegar a entorpecerla. Son las cláusulas clásicas que preservan la aplicación de los anteriores o ulteriores tratados entre las mismas partes y en la misma materia, en tanto beneficien la cooperación, con lo que el panorama normativo convencional se torna más complejo, al necesitar los operadores de la justicia, conocer y decidir, cual fuente resulta aplicable a determinado caso que se le plantee ${ }^{10}$.

\section{d) Extensión Territorial}

Muchos de los países de la región tienen una gran extensión territorial y ello provoca que los justiciables y los aplicadores de convenios estén muy lejos de los centros de tramitación de los pedidos y de las autoridades centrales o coordinadoras de aplicación de los convenios. Esto se traduce en la dificultad de conocer, en muchos casos, la totalidad de los convenios o mecanismos vigentes sobre una materia y las bondades de utilizar uno u otro instrumento. Ello también genera quejas por el retardo para la solución de los casos en materia penal que, usualmente requieren un accionar sin demoras, especialmente en situaciones especialmente en materia de criminalidad compleja que involucran trata de menores o drogas. Ello finalmente origina reclamos de comunicación directa entre ellos -jueces, fiscales y operadoresespecialmente en zonas de frontera, a fin de evitar la burocracia de las solicitudes a través de autoridades citas en las capitales.

\section{e) Falta de medios}

En muchos casos la falta de recursos es un tema que dificulta, tanto el conocimiento de las fuentes aplicables como la existencia de nuevas tecnologías o redes, que coadyuven a hacer posible la vinculación con otros operadores o actores de la cooperación a la hora de resolver casos transfronterizos.

Otra dificultad surge respecto del mantenimiento económico de las plataformas informáticas lo que provoca que sistemas que fueron de gran ayuda en su momento como lo fue el mecanismo "Groove de la OEA" haya perdido relevancia por no encontrar recursos para su mantenimiento y actualización adecuada.

En el caso de la IberRed (Red Iberoamericana de Cooperación Jurídica) y su correo electrónico seguro (Iber@), a través de ambos se instituye un mecanismo de contactos informales previos para la tramitación transmisión de solicitudes de cooperación entre autoridades centrales.

Otro tema no menor lo representa, la estadística de casos de

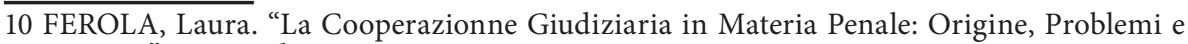
Prospettive”. Rivista di Diritto Europeo, 1999, n 1. 
derecho internacional, ya que el tiempo promedio de solución de casos complejos, resultan cruciales a la hora de instrumentar bancos de datos regionales o en la necesidad de instalar nuevas tecnologías de comunicación - correos electrónicos seguros o equipos de video conferencia.

\section{f) Falta de coordinación y control}

A la dispersión de recursos logísticos, se suma el desafío de la coordinación entre las instituciones encargadas de la puesta en marcha de nuevos convenios de cooperación. Así se hace imprescindible la colaboración entre ellos, principalmente entre Autoridades Centrales, Judiciales y Defensorías -nacionales y provinciales o estaduales-, por un lado y, las de estos con los operadores de la justicia, por el otro.

\section{LOS FOROS PRODUCTORES DE NORMAS Y EL DERECHO INTERNACIONAL PRIVADO EN LA BÚSQUEDA DE LA EFECTIVIDAD}

Uno de los ámbitos de trabajo para encontrar respuestas a los desafíos y procurar lograr dar soluciones a las problemáticas que se presentan es el de la actividad desarrollada en los foros productores de normas y de las organizaciones intergubernamentales quienes vienen estudiando el tema en profundidad y ensayando e instrumentando prácticas novedosas y diferentes mecanismos tecnológicos, tanto de "hard law" como de "soft law" que coadyuven a mejorar la efectividad de las soluciones en materia de lucha contra la criminalidad.

Solo por citar algunos de ellos, enumeraremos a los que han generado algunas prácticas y herramientas novedosas en sus respectivos espacios de desarrollo en el ámbito del Mercosur; y de otros organismos intergubernamentales en los que el MERCOSUR está inserto, como por ejemplo la OEA; la Conferencia de Ministros de Justicia de Iberoamérica; o la UNODC de Naciones Unidas.

A continuación se pasará revista a alguna de esas buenas prácticas promovidas e instrumentadas.

\section{a) Reunión de Ministros de Justicia del MERCOSUR}

Es el foro que dentro de la estructura institucional del Mercosur tiene dentro de su cometido avanzar hacia la armonización legislativa en materias de cooperación jurídica y generar los instrumentos $\mathrm{y}$ mecanismos de la asistencia jurídica internacional que el proceso de integración le demande ${ }^{11}$. En ese ámbito son varios los instrumentos

11 DREYZIN de KLOR, Adriana. "El MERCOSUR. Generador de una fuente de Derecho Internacional Privado". Buenos Aires: Zavalía Editores, 1997. DREYZIN de KLOR, Adriana. 
de cooperación jurídica que se han generado y que regulan en muchos casos cuestiones relacionadas con la cooperación penal. ${ }^{12}$

\section{b) CIDIP-OEA}

Es sabido que dentro del esquema interamericano, coexisten varios convenios que alcanzan dimensión continental y que han generado, por una parte, una gran cantidad de normativa en diferentes materias y aspectos relacionados con la temática que nos ocupa y que contribuye al entramado de fuentes aplicables sobre temas específicos, en la medida que muchos Estados de la región han ratificado o adherido a sus Convenciones,

\section{c) La Conferencia de Ministros de Justicia de los Países Iberoamericanos}

Se trata de una organización intergubernamental compuesta por los 22 Estados que comprenden Iberoamérica (España, Portugal, Centro y Sudamérica). En el marco de esta organización se han venido celebrando convenios de cooperación jurídica en materia penal, especialmente en materia de las problemáticas procesales en torno a la criminalidad compleja, que proponen armonizar o unificar soluciones entre los países de la región, dadas sus características comunes de historia y lenguaje $\mathrm{e}^{13}$.

Mención especial merece la actividad informal desarrollada por la IberREd a través de sus Puntos de Enlace, que aglutina la actividad de Cortes y Superiores Tribunales de Justicia de los Estados, Ministerios Públicos y Ministerios de Justicia y Autoridades Centrales.

\section{HACIA LA BÚSQUEDA DE SOLUCIONES Y RESPUESTA EFECTIVAS}

Una vez reseñadas las diferentes dificultades que presenta la cooperación en el espacio mercosureño y los consiguientes desafíos a encarar, se volverá a esquematizar acerca de los desafíos que presenta la región en la materia para ir dando algunas de las respuestas que vienen aportando los Estados en el marco del contexto de integración regional que nos ocupa, con miras a demostrar el camino recorrido para la efectividad de las soluciones que se pueden considerar.

\footnotetext{
"Hacia el ordenamiento jurídico del MERCOSUR en La Ley To1996-C", Buenos Aires, 1996, p. 1189-1201.

12 CARBAJAlES, M. "La armonización legislativa". En Revista de Derecho del Mercosur. 1998, n 4, año 2, p. 161-167.

13 Disponible: <www.comjib.org.es>
} 


\begin{tabular}{|l|l|}
\hline \multicolumn{1}{|c|}{ Desafío } & \multicolumn{1}{c|}{ Propuesta } \\
\hline $\begin{array}{l}\text { Proliferación de Fuentes Convencionales } \\
\text { sobre mismos temas entre mismas } \\
\text { Partes. }\end{array}$ & $\begin{array}{l}\text { a) En la nueva normativa de cooperación } \\
\text { se fije la vigencia del convenio respecto } \\
\text { de los otros; b) en los ya existentes, } \\
\text { se establezcan prelaciones y guías } \\
\text { orientativas para los operadores. }\end{array}$ \\
\hline Cláusulas en beneficio de la cooperación & $\begin{array}{l}\text { Elaboración de Guías y Manuales que } \\
\text { orienten sobre la mejor fuente a aplicar } \\
\text { basada en su eficiencia. }\end{array}$ \\
\hline $\begin{array}{l}\text { Interconexión entre operadores de } \\
\text { justicia }\end{array}$ & $\begin{array}{l}\text { Fomento de su inter-relacionamiento a } \\
\text { través de autoridades coordinadoras y } \\
\text { medios electrónicos. }\end{array}$ \\
\hline $\begin{array}{l}\text { Necesidad de Bancos de Datos e } \\
\text { Intercambio de Información. }\end{array}$ & $\begin{array}{l}\text { Cruzado de datos entre los sectores que } \\
\text { registran distintos de información. }\end{array}$ \\
\hline Extensión Territorial de los países & $\begin{array}{l}\text { Utilización de los medios electrónicos } \\
\text { y cursos de difusión y en el interior de } \\
\text { los respectivos países, a través de redes } \\
\text { sociales. }\end{array}$ \\
\hline Recursos presupuestarios & $\begin{array}{l}\text { Optimización de los existentes mediante } \\
\text { la coordinación de sectores y asignación } \\
\text { de partidas para la informatización de los } \\
\text { sectores involucrados. }\end{array}$ \\
\hline
\end{tabular}

A continuación, entonces, se desarrollará el contenido de las iniciativas en curso o a instrumentar para encarar los desafíos.

\section{a) Comunicación entre Autoridades:}

Este sistema propicia una alternativa a las autoridades centrales y tiene como fin el permitir la directa comunicación entre jueces y operadores. Sin embargo, esta alternativa, con la salvedad explícita de la cooperación entre autoridades fronterizas, parece que desde la perspectiva de nuestra región, debería ser incorporada en forma gradual y experimental, para tomarla como ejemplo piloto desde lo nacional y evaluar su extensión a otros países de la región, hasta tanto se salven los obstáculos que aún subsisten, para su aplicación.

Por ejemplo, la imposibilidad en algunos países para permitir la comunicación directa entre magistrados y defensores -aún entre los de zonas de frontera- sin que se pase a través de las autoridades centrales ubicadas en las capitales.

La relevancia radica principalmente en la falta de difusión de muchos de los nuevos convenios y modalidades de la cooperación por parte de operadores en territorios muy dilatados o de difícil comunicación. 
Entretanto, se torna necesario un punto común de contacto entre las autoridades de los diferentes Estados y dentro de cada uno de estos, con los respectivos organismos encargados de la aplicación del derecho.

La utilización de las TICs, (técnicas de la información y la comunicación) puede representar una gran ayuda para el diligenciamiento de soluciones "on line" o la descentralización de autoridades coordinadoras en Estados provincias y municipios como parte de buenas prácticas aportadas.

Como ejemplo se puede citar a la autoridad instaurada en el Tribunal Supremo de Justicia de la Provincia de Mendoza, en la Argentina.

\section{b) Vinculación de redes e instituciones. El papel de las Autoridades Centrales y Coordinadoras}

Es menester destacar la importancia que reviste el realizar encuentros conjuntos entre los diferentes actores jurídicos encargados de la aplicación de los convenios de cooperación. Así, intercambios de experiencias y encuentros conjuntos entre jueces, defensores y operadores de la justicia en general, será un muy buen punto de partida para la difusión de herramientas, intercambio de información y pautas para la aplicación de prácticas y tecnología moderna que permita agilizar la cooperación.

\section{c) Las buenas prácticas y las nuevas tecnologías}

Pueden señalarse también como iniciativas auspiciosas de cooperación en la materia, la constitución de redes y técnicas desarrolladas en la región y que han tomado algún referente en lo ya ensayado en el viejo continente. Entre ellos, por ejemplo, la "IberRed" en el ámbito iberoamericano, inspirada, a su vez, en la "Red Judicial Europea". Esta "Red" es, como lo dice su nombre, una red de contactos entre autoridades encargadas de la aplicación de justicia entre los países del bloque iberoamericano, que comprende a los Ministerios de Justicia de los Estados Parte, a las Autoridades Centrales, al Ministerio Público y el Poder Judicial ${ }^{14}$.

Párrafo aparte merece la creación de la "Red Interamericana de Cooperación Jurídica" Groove, en el marco de la OEA, que ha alcanzado importantes logros a través del funcionamiento del correo electrónico seguro con firma encriptada "Groove" para la trasmisión y diligenciamiento de las solicitudes de cooperación en la materia.

Con el mismo espíritu en el ámbito de la Reunión de Ministros de

14 Eser y Lodogny. Principles and Procedures for a new Transnational Criminal Law. Freiburg in Brisgau: Max-Planck-Institut, 1992. 
Justicia del Mercosur, se creó el "Foro de Autoridades Centrales", que se reúne periódicamente con el fin de detectar las bondades y dificultades que genera la aplicación de los Protocolos y Acuerdos aprobados en su seno y en la inter vinculación de dichos instrumentos jurídicos con otros pre existentes o que se pongan en marcha, sobre los mismos temas entre las mismas partes

Finalmente, en el marco de Naciones Unidas, más específicamente en la UNODC, se han desarrollado diferentes "Guías" y "Manuales" de Buenas Prácticas que se ocupan de resolver las diferentes dificultades que plantea la aplicación de sus Convenios en materia de cooperación penal y criminalidad compleja.

\section{d) Encuentros y Tareas de Difusión}

La difusión de convenios, mecanismos y herramientas resulta esencial. En ese marco de acción se han propuesto distintos caminos, desde la identificación de elementos comunes y posturas de consenso en el marco regional mediante esquemas comparativos de soluciones normativas en el marco de la Reunión de Ministros de Justicia del MERCOSUR, como la elaboración de guías orientativas o leyes modelo que tomaran en cuenta los caracteres comunes de cada sistema nacional, para que pudieran luego ser trasladadas a su normativa interna en momentos de tornar operativas las normas convencionales adoptadas.

\section{CONCLUSIONES}

Como corolario del panorama presentado, las dificultades detectadas y los consiguientes desafíos sobrevinientes en el marco del MERCOSUR y de este espacio en su inserción en los sistemas interamericano e iberoamericano, cabe concluir que a la actividad desarrollada por los diferentes foros integrantes del sistema institucional mercosureño, tienden a la promoción de normas de cooperación armonizadas, a lo que se le pueden sumar diferentes propuestas que se han planteado para agilizar los pedidos de cooperación que tiene que ver con buenas prácticas o utilización de tecnologías manifestados en redes, correos seguros y encriptados o plataforma de sustentación para el desarrollo de solicitudes.

En materia de buenas prácticas, la actividad de puntos de contacto, enlaces o autoridades centrales ha demostrado también ser una buena alternativa para enfrentar los desafíos en la materia.

Para finalizar solo restaría destacar la importancia de la difusión a desarrollar entre los operadores de la justicia, acerca de la existencia de herramientas disponibles y propuestas a implementar para facilitar y agilizar la cooperación penal internacional. 
En virtud de lo manifestado entonces se puede pasar revista al impacto de las soluciones instrumentadas o a implementar:

\section{a) Armonización y tipificación de figuras}

Ante la disparidad en la tipificación de las figuras delictuales en la fuente interna de cada país - por ejemplo, "terrorismo", "lavado de activos" o "trata de personas"- se propone encarar una tarea de armonización conceptual como modelo, basada en características y elementos comunes a cada realidad nacional, para luego tomarlos como parámetros de legislación en la materia en cada nueva norma nacional que se dicte en los Estados, tanto en lo que se refiere a lo procesal como a la sustancial. Esos parámetros conceptuales de referencia, tendrán que armonizarse también con las penalidades asignadas a cada figura.

En esa tarea se debería procurar también la elaboración de normas operativas que tornen efectivos los numerosos convenios internacionales de cooperación que se han dictado y que tienen, en su mayoría normas programáticas. Asimismo, se propone se elaboren guías y manuales de buenas prácticas que demuestren a los operadores de la justicia el tratamiento que se le da a ciertas figuras, sin perjuicio de la definición del tipo legal que se le asigne. Es el caso, por ejemplo, del delito de "trata de personas", en que varias de sus facetas están tratadas en las legislaciones domésticas pero que no responden al nombre de trata, sino identificado como delito "contra la integridad sexual".

Otra manifestación en materia de armonización, que se aporta, es la idea de canalizar en convenios regionales operativos la normativa de convenciones más generales de índole programática.

\section{b) Superposición normativa y cláusulas en beneficio de la cooperación}

Para intentar resolver el tema de la gran cantidad de fuentes vigentes sobre las mismas materias y su compatibilidad con las nuevas que se elaboren entre las mismas Partes y sobre los mismos temas, se propone incorporar entre las "Disposiciones Generales" de los nuevos convenios de cooperación que se concluyan, cláusulas por la que la entrada en vigencia de los nuevos convenios vaya derogando los anteriores que se ocupen de esos mismos temas.

Por su parte, en lo que hace a los convenios ya vigentes, se impone realizar una tarea de sistematización de fuentes convencionales en base a la efectividad probada en su aplicación. En ese camino se puede fijar -si no se quiere derogar o denunciar- un orden de prelación regional convenido entre los operadores del derecho de un espacio común. Para ese resultado se impone efectuar una amplia convocatoria abierta a la 
participación de jueces, fiscales, defensores, consejos de magistratura, Ministerios de Justicia, autoridades centrales de aplicación de convenios, académicos, empresarios y profesionales, quienes vuelquen sus inquietudes y acuerden guías de facilitación de la aplicación de convenios a nivel regional.

\section{c) Cláusulas de “orden público" y "seguridad nacional”.}

Ante los escollos que se han detectado por la inclusión de cláusulas de salvaguarda del orden público o de los intereses superiores del Estado, a la hora de combatir la criminalidad compleja en los espacios integrados, se propone redactar una carta fundamental común para la región o una convención que recoja principios uniformes de salvaguarda, limitados por el respeto a los derechos fundamentales y humanos vigentes en todo el espacio regional.

\section{d) Reconocimiento de sentencias y extradición:}

Se reconoce necesaria la búsqueda de mecanismos más modernos que permitan facilitar la extradición y el reconocimiento de sentencias en materias de interés común. Como punto de partida se deberá comenzar con la promoción de investigaciones de entreayuda para casos de crímenes transfronterizos graves, particularmente para los casos de crimen organizado, más específicamente la posibilidad de formar equipos conjuntos de tareas- tal como lo prescribe la Convención de la ONU contra la Delincuencia Organizada y siguiendo la tarea desarrollada en la materia en el ámbito del MERCOSUR.

Por otra parte, algunos de los convenios celebrados ya prevén la cooperación directa entre jueces de zonas de frontera. Dicha comunicación puede entonces, extenderse en lo interno, a la colaboración a prestar entre otras autoridades o sectores para contribuir al esclarecimiento de este tipo de delitos -aduanas, registros civiles, destacamentos de frontera, áreas de control integrado. Respecto a su instrumentación en los otros países que tienen algún obstáculo para su implementación, puede proponérseles para salvar esos obstáculos tomar en consideración la calidad de una normativa común en beneficio de la integración o basada en la ciudadanía única que detentan los habitantes de un mismo espacio regional y la consiguiente igualdad de trato a recibir entre ellos.

En el mismo sentido, se hace necesario avanzar aún más en el reconocimiento mutuo de los medios de prueba en los diferentes países y ayudarlos con la más rápida comunicación que trae aparejada la incorporación de las nuevas tecnologías, por ejemplo utilizando la videoconferencia. 
Otra alternativa puede ser la del establecimiento de un sistema más ágil para la detención de delincuentes, estableciendo, sobre las pautas del convenio de cooperación policial regional, suscribiendo acuerdos interinstitucionales que permitan operaciones conjuntas para el arresto de delincuentes sobre bases de cooperación entre fuerzas para todos los Estados de la región, simplificando los requisitos para la detención y entrega.

\section{e) Interpretación armónica}

Ante la falta de un tribunal regional que interprete en forma unívoca la normativa en la materia y que instrumente y supervise un accionar conjunto para combatir la criminalidad que se presente a nivel transnacional, se hace necesaria la existencia de una interpretación unívoca para las autoridades judiciales, el ministerio público, las autoridades policiales y las fuerzas de seguridad.

Con los aportes y propuestas desarrolladas en los apartados precedentes se pretende proponer como aporte una serie de buenas prácticas para facilitar y agilizar la cooperación, utilizando las nuevas tecnologías y los medios electrónicos sin que ello vaya reñido con la necesaria seguridad jurídica que debe imperar en la asistencia en general y más aún en la materia penal.

\section{REFERENCIAS BIBLIOGRÁFICAS}

BAIGÚN, David. "El abogado y las buenas causas". Revista del Colegio Público de Abogados de la Capital Federal, n 84.

BOGGIANO, Antonio. Derecho Penal Internacional. Buenos Aires: Lexis Nexis- Abeledo Perrot, 2003.

CERVINI, Raúl. "La Cooperación Judicial Penal Internacional: Concepto y Proyección". Ponencia en Curso de Cooperación Penal Internacional. Rio de Janeiro: Carlos Alvarez Editor, 1994.

GREGORIO GARZÓN, Clariana. Sobre la Noción de Cooperación en el Derecho Internacional. Revista Española de Derecho Internacional. 1976, n 1, p. 33.

DE CASTELLO CRUZ, Luis. "Informe del Brasil. Cooperación Interamericana en los Procedimientos Penales". Revista Universidad Nacional Autónoma de México. México. D.F., 1983.

DE PAVÍA, Agustín. "Asistencia Judicial Internacional en materia Penal". Ponencia presentada ante el II Seminario Internacional sobre Cartagena de Indias, Colombia, febrero de 2003.

DREYZIN DE KLOR, Adriana. El MERCOSUR y sus Dimensiones. Buenos Aires: Editorial Zavalía, 1997. 
ESER, Lodogny. Principles and Procedures for a new Transnational Criminal Law. Freiburg in Brisgau, 1992.

FEROLA, Laura. "La Cooperazionne Giudiziaria in Materia Penale: Origine, Problemi e Prospettive". Rivista di Diritto Europeo. 1999, n 1.

GARCÍA, Luis M. "Auxilio Judicial Internacional y Soberanía Estatal. Problemas que plantea los principios de la validez material del derecho penal: jurisdicción concurrente y múltiple persecución penal)". Revista La Ley 1992-B. P. 928.

GARZÓN CLARIANA, Gregorio. "Sobre la Noción de Cooperación en el Derecho Internacional", Revista Española de Derecho Internacional. $\mathrm{N}^{\circ} 1,1976$, p. 33.

HARDING, Christopher. "Exploring the intersection of European law and national criminal law". European Law Review. 2000, vol 25/4 Aug. 2000 .

JAYME, Erik. "Identité Culturelle e Intégration: le Droit International Privé Posmoderne". Recueil de Cours. 1995, tomo 251, p. 37

MOYA DOMÍNGUEZ, María Teresa. “El MERCOSUR y la Integración”. Revista Colegio Público de Abogados. 2003.

PIOMBO, Horacio. Tratado sobre Extradición. Buenos Aires: Editorial Depalma, 1998.

SIEBER, Ulrich. "Delitos Informáticos y otros Delitos contra la Tecnología de la Información”. Publicación Max Planck Institut. Friburg. Traducción del original en inglés por el Dr. Eugenio Zaffaroni.

SLOKAR, Alejandro. "Criminalidad Compleja"- Terrorismo y Cybercriminalidad. Revista de la Subsecretaría de Política Criminal. Ministerio de Justicia y Derechos Humanos. 2004. p. 35.

\section{RESUMEN BIOGRÁFICO}

Juan José Cerdeira es Abogado por la UBA. Doctor en Derecho Internacional (UNA). Profesor de Derecho Internacional Privado y de Derecho de la Integración, en diferentes Universidades Públicas y privadas en la Argentina y en el MERCOSUR. Investigador de la UNL en Argentina. Autor de diferentes artículos sobre integración. Negociador en el MERCOSUR otros foros internacionales. 